\title{
Endoscopic diagnosis and treatment of precancerous colorectal lesions in patients with inflammatory bowel disease: How does the latest SCENIC international consensus intersect with our clinical practice?
}

\author{
Dong Wu, Jing-Nan Li, Jia-Ming Qian \\ Department of Gastroenterology, Peking Union Medical College Hospital, \\ Chinese Academy of Medical Sciences, Beijing 100730, China
}

Address for Correspondence: Professor Jing-Nan Li, Department of Gastroenterology, Peking Union Medical College Hospital, Chinese Academy of Medical Sciences, Beijing 100730, China. Email: lijn2008@126.com

\begin{tabular}{|l|}
\hline Access this article online \\
Website: \\
www.intern-med.com \\
DOI: \\
10.1515/jtim-2017-0008 \\
\hline Quick Response Code: \\
\hline \\
\end{tabular}

Inflammatory bowel disease (IBD) refers to a group of chronic non-specific inflammatory conditions of the gastrointestinal tract, and is particularly represented by ulcerative colitis (UC) and Crohn's disease (CD). In recent years, the incidence of IBD appears to remain stable in the Western hemisphere, but in China, it shows a rapidly growing trend due to the rapid industrialization, urbanization and westernized lifestyle. An epidemiological survey by Peking Union Medical College Hospital in Daqing, China showed that the incidence of UC is 1.64 per 100000 and for CD, it is 0.13 per $100000 .^{[1]}$ Although the absolute incidence of IBD is not currently disturbing, the great number of patients with IBD in this country has added up to a concerning burden, because the disease is usually liable to relapse, hardly curable, and may last a long course. Based on the characteristics and the increasing incidence of IBD, it is estimated that in the near future, IBD will become a common gastrointestinal disorder in China.

IBD patients face a significantly increased risk of colorectal cancer (CRC) over time. Long-term follow-ups indicate that the 10-, 20- and 30-year incidence rates of CRC in patients with UC are $2 \%, 8 \%$ and $18 \%$, respectively, significantly higher than those in the general population. ${ }^{[2]}$ When $\mathrm{CD}$ involves $1 / 3$ of the colon, the risk of colorectal cancer is similar to that of UC. ${ }^{[3]}$ Therefore, for IBD patients with a long disease duration, timely colonoscopic screening should be prompted for detecting
CRC and precancerous lesions early, and thereby improving prognosis. However, compared with the general population, CRC screening with classical colonoscopy is relatively difficult in IBD patients, mainly because most of the precancerous lesions in IBD patients are flat in shape (type IIa or lateral growth type) and distributed against a backdrop of mucosa with varying degrees of edema, congestion, erosion, and scar formation, which often results in misdiagnosis unless in skilful hands.

To minimize the drawbacks of endoscopic studies for diagnostics, in 2010, the American College of Gastroenterology (ACG) recommended random biopsy during colonoscopy in patients with IBD. For random biopsy, four-quadrant random biopsies were obtained from each 10 $\mathrm{cm}$ interval during colonoscopy, and a minimum of 32 biopsies are thus collected from each patient to detect dysplasia. ${ }^{[4]}$ Although this method modestly improves the detection rate, it inevitably results in many unnecessary biopsies. Therefore, the ACG recommendation is not only inefficient for problem-solving, but also represents a waste of health resources. In recent years, the rapid advancement in endoscopic technology and ever-increasing use of highresolution endoscopy, chromoendoscopy, image-enhanced endoscopy and magnifying endoscopy have greatly enhanced the detection of precancerous lesions in IBD patients. Since 2013, high-resolution chromocolonoscopy has been used to 
screen precancerous lesions in IBD patients in Peking Union Medical College Hospital. Up to now, we have screened 95 IBD patients with a disease duration $\geq 5$ years and identified one case of cancer, two cases of high-grade dysplasia, and 6 cases of low-grade dysplasia. We noted that most of the IBD-related dysplasia were detectable with the new-generation endoscopic technology and precise biopsy. ${ }^{[5-6]}$ The latter refers to a newly introduced technique, termed as targeted biopsy, which is more efficient as compared to the classical random biopsy. In 2015, the latest SCENIC consensus on surveillance and management of dysplasia in inflammatory bowel disease was simultaneously published in two reputed journals, the Gastroenterology and the Gastrointestinal Endoscopy. ${ }^{[7]}$ Compared with the 2010 edition, the updated document throws light on the research progress in recent years and incorporates the latest understanding in this field. For example, regarding terminology, due to the significant improvement in endoscopic diagnostics, the SCENIC consensus recommends that the term 'dysplasia associated lesion or mass' (DALM) should no longer be used. Previously, limited resolution of classical endoscopy greatly compromised the visualization of mucosal surface details, and therefore, endoscopists had to rely on low-efficiency methods such as random biopsy to detect dysplasia. In this context, DALM was used to describe the colonic mucosa containing precancerous lesions. The rapid development of endoscopic techniques in recent years has enabled us to fully observe suspicious lesions and to determine the nature of the lesion with an accuracy very close to that of histological diagnosis. Therefore, DALM, as a relatively vague term, should be discarded.

Given that endoscopic screening has not been in widespread use in Chinese patients with a long disease duration of IBD, the SCENIC consensus stands for a highly valuable reference for gastroenterologists and endoscopists in China. In this regard, we present a brief interpretation of this consensus based on our clinical practice and expertise.

\section{HIGH-RESOLUTION}

\section{CHROMOENDOSCOPY IS THE GOLD} STANDARD FOR SCREENING IBDRELATED CRC AND PRECANCEROUS LESIONS

Indigo carmine staining was initially used for the endoscopic diagnosis of early gastric cancers, and subsequently, applied to the diagnosis of diseases in the digestive tract. Using high-resolution colonoscopy combined with indigo carmine (or methylene blue), staining to visualize the colonic mucosa is currently the first-line screening method in IBD patients. A study showed that dysplastic lesions detected by high-resolution endoscopy (HD 1080i system) is 2.2 (95\%CI: 1.1-4.5) times the cases identified by classical endoscopy (SD480 system). ${ }^{[8]}$ On the basis of high-resolution endoscopy, the application of staining can further improve the lesion detection rate. According to the new SCENIC consensus, the detection rate of IBD-related dysplasia by chromoendoscopy is 1.8 (95\% CI: 1.2-2.6) times as that by the white light endoscopy, corresponding to an absolute increase in detection rate by 6\% (95\% CI: 0-9). ${ }^{[7]}$

In clinical practice, we usually perform screening when IBD patients are clinically stable, and thus, they may have less active mucosal inflammation that would interfere with the endoscopic investigation. During the procedure, $0.03 \%$ indigo carmine is sprayed evenly onto the colonic mucosa along which the passage of colonoscope being withdrawn after it has been previously advanced to the ileocecal junction. This can clearly show the subtle elevated or depressed changes on the mucosal surface and facilitate the detection of adenoma and dysplasia (especially flat or depressed lesions). If a suspicious lesion is found, a higher concentration of indigo carmine $(0.13 \%)$ can be sprayed to reveal its outline and boundaries and determine its nature. According to our experience, although spraying indigo carmine is somewhat time-consuming (say, a mean increase in total operation time by $\sim 10 \mathrm{~min}$ ), it dramatically improves the resolution in revealing the diseased mucosa. Given that indigo carmine is inexpensive and the staining manipulation is relatively simple, this screening method can be suitable for widespread use in China.

\section{NARROW BAND IMAGING (NBI) IS NOT RECOMMENDED}

In the past decade, many types of image-enhanced endoscopy have been increasingly used in clinical practice, which include NBI, i-SCAN and Fuji intelligent chromoendoscopy. The working principle of imageenhanced endoscopy is the use of spectrophotometry to generate light sources of different wavelengths to highlight the minute structure and microvasculature of the mucosa. Such technology, also known as 'electronic staining', can achieve rapid switching via the operating portion of endoscope, thereby averting the inconvenience of staining sprays and increasing the efficiency of examination. For instance, with NBI, a filter is used to narrow the white light spectrum with a range of $30 \mathrm{~nm}$, thus obtaining a blue light at $415 \mathrm{~nm}$ and a green light at $540 \mathrm{~nm}$, both of which can highlight the microvasculature of the mucosa. The value of the electronic staining in the diagnosis of CRC has been confirmed in the general population. However, so far, there has been no evidence demonstrating that NBI is better than chromoendoscopy in the detection and diagnosis of IBD- 
related dysplasia. ${ }^{[9-10]}$ Because of insufficient brightness and relatively dim vision, it is difficult to use classical NBI to observe the mucosa when colonoscope is withdrawn. Although the new-generation NBI system offers brighter vision, still this doesn't mean that it is superior to the chromoendoscopy in screening IBD-related dysplasia. ${ }^{[1]}$ The reason is that the feature of NBI is to highlight blood vessels (dark brown or dark), while it has no advantage in displaying the subtle morphological alterations of glands. In IBD patients, due to repeated cycles of bowel mucosal inflammatory damage and repair, the microvascular structures usually appear significantly aberrant from normal anatomy and sometimes even diminish. In these cases, NBI demonstrates a poor performance as compared to chromoendoscopy most of the time.

Our experience also suggests a great challenge to detect dysplasia by NBI alone. However, when suspicious lesions are found through white light or staining, NBI is helpful to differentiate between benignity and malignancy by visualizing the microvascular morphology of the mucosa.

\section{PROPER DETERMINATION OF INDICATIONS FOR ENDOSCOPIC TREATMENT}

Accurate diagnosis should prelude a rational treatment. Once a lesion is found by endoscopy, the endoscopists should study the morphology of the lesion carefully, estimate the histologic type, and obtain biopsies when necessary to determine the nature of the lesion. Generally, a red pedunculated lesion with a cap of adherent mucus but without a clear gland structure on the surface is an inflammatory pseudopolyp or a hyperplastic polyp. Unless these lesions have complications such as bleeding, they may not necessitate resection but should be followed up on a regular basis. It should be pointed out that due to the impact of chronic inflammation on the mucosa, Kudo's pit pattern classification is not very suitable for the judgment of IBD-related dysplasia.. ${ }^{[12]}$ So far, the prediction of histological types of mucosal lesions in IBD patients based on the morphological features under endoscopy remains to be further studied.

Due to the rapid advances in endoscopic treatment, endoscopic polyp resection has become a routine procedure. In the past, once an IBD patient was found to have high-grade dysplasia or cancer, total proctocolectomy should be performed. If an ileoanal pouch surgery seemed technically infeasible, a permanent ileostomy was to be selected. This would greatly affect the patient's quality of life. Currently, it is advocated that if the cancerous lesion is limited to the mucosa or invades the submucosal layer $\leq 1000 \mu \mathrm{m}$ (SM1), the risk of lymph node metastasis is lower and most patients are eligible for endoscopic resection. While regular follow-up examination after endoscopic resection is mandatory, there is no consensus on the interval for follow-ups. Generally, the interval should be shorter than as for average patients (e.g., 3-6 months). For cancerous lesions that invade the submucosal layer $>1000 \mu \mathrm{m}$, with no clear-demarcated boundaries, or cannot be removed endoscopically, open surgery should be considered.

In conclusion, the incidence of IBD in China is on the rise. For patients with a long disease duration of IBD, the risk of canceration should not be under-estimated. A combination of high-resolution endoscopy, staining screening and precise biopsy would help detect more precancerous lesions. Following an accurate diagnosis, reasonable selection of minimally invasive treatment options such as endoscopic resection will improve the prognosis and quality of life in patients with IBD.

\section{Conflict of Interest}

None declared.

\section{REFERENCES}

1. Yang H, Li YM, Wu W, Sun QW, Zhang YZ,Zhao W, et al. The incidence of inflammatory bowel disease in Northern China: a prospective population-based study. PLoS One 2014; 9: e101296.

2. Eaden JA, Abrams KR, Mayberry JF. The risk of colorectal cancer in ulcerative colitis: a meta-analysis. Gut 2001; 48: 526-35.

3. Canavan C, Abrams KR, Mayberry J. Meta-analysis: colorectal and small bowel cancer risk in patients with Crohn's disease. Aliment Pharmacol Ther 2006; 23: 1097-104.

4. Farraye FA, Odze RD, Eaden J, Itzkowitz SH, McCabe RP, Dassopoulos T, et al. AGA medical position statement on the diagnosis and management of neoplasia in inflammatory bowel disease. Gastroenterology 2010; 138: 738-45.

5. Rutter MD, Saunders BP, Wilkinson KH, Kamm MA, Williams CB, Forbes A. Most dysplasia in ulcerative colitis is visible at colonoscopy. Gastrointest Endosc 2004; 60: 334-9.

6. Rubin DT, Rothe JA, Hetzel JT, Cohen RD, Hanauer SB. Are dysplasia and colorectal cancer endoscopically visible in patients with ulcerative colitis? Gastrointest Endosc 2007; 65: 998-1004.

7. Laine L, Kaltenbach T, Barkun A, McQuaid KR, Subramanian V, Soetikno R. SCENIC international consensus statement on surveillance and management of dysplasia in inflammatory bowel disease. Gastroenterology 2015; 148: 639-51.

8. Subramanian V, Ramappa V, Telakis E, Mannath J, Jawhari AU, Hawkey CJ, et al. Comparison of high definition with standard white light endoscopy for detection of dysplastic lesions during surveillance colonoscopy in patients with colonic inflammatory bowel disease. Inflamm Bowel Dis 2013; 19: 350-5.

9. Ignjatovic A, East JE, Subramanian V, Suzuki N, Guenther T, Palmer $\mathrm{N}$, et al. Narrow band imaging for detection of dysplasia in colitis: a randomized controlled trial. Am J Gastroenterol 2012; 107: 885-90.

10. Pellisé M, López-Cerón M, Rodríguez de Miguel C, Jimeno M, Zabalza $\mathrm{M}$, Ricart E, et al. Narrow-band imaging as an alternative to chromoen- 
Wu et al.: Endoscopic diagnosis and treatment of precancerous colorectal lesions in IBD patients

doscopy for the detection of dysplasia in long-standing inflammatory bowel disease: a prospective, randomized, crossover study. Gastrointest Endosc 2011; 74: 840-8.

11. Efthymiou M, Allen PB, Taylor AC, Desmond PV, Jayasakera C, De Cruz $\mathrm{P}$, et al. Chromoendoscopy versus narrow band imaging for colonic surveillance in inflammatory bowel disease. Inflamm Bowel Dis 2013; 19: 2132-8.
12. Hata KI, Watanabe T, Motoi T, Nagawa H. Pitfalls of pit pattern diagnosis in ulcerative colitis-associated dysplasia. Gastroenterology 2004; 126 : 374-6.

How to cite this article: Wu D, Li JN, Qian JM. Endoscopic diagnosis and treatment of precancerous colorectal lesions in patients with inflammatory bowel disease: How does the latest SCENIC international consensus intersect with our clinical practice?. J Transl Intern Med 2017; 5: 4-7. 